\title{
Modular Lower Extremity Prosthetics
}

\author{
J. FOORT,
}

formerly. Technical Direcior Maniroba Rehabilitarion Hospital

Prosthetics and Orthotics Research and Development Unit, Winnipeg, Canada.

A new term is entering prosthetics. The term is "Modular". A modular prosthesis is one which can be assembled to a high degree from prefabricated parts and can be adjusted on the amputee very rapidly to bring it to optimum function. Clinical services at the Manitoba Rehabilitation Hospital in Winnipeg, Canada, were the first to employ modular prosthetics on a routine basis and this has been going on since 1965. Development of the modular system for lower extremity amputees was carried out on an evolutionary basis using what engineers had learned in the design and clinical testing of devices of this sort prior to 1965. The Winnipeg system used not only currently available hardware, however, but hardware designed in Winnipeg to fill in the gaps which blocked use of modular prosthetics up until then.

The aim was to bring into being a comprehensive system for lower extremity amputees which would utilize existing components and introduce what new designs were necessary to make the modular system work clinically. Design objectives were to develop needed components so that a bare minimum would be required. The evolutionary process used to develop the system was based on meeting any of the needs in the rehabilitation system which would reduce delays and interruptions to rehabilitation programmes even if usefulness were limited to only parts of the programmes. Thus, in the beginning, prefabricated sockets were used with conventional temporary prostheses so that both conditioning of the stump and training on a prosthesis could be carried out simultaneously rather than sequentially as was previously the case. At first, sockets for above the knee amputees were put into service. Four sockets left and right were used, these being adjustable in girth and varying in size so that as stump maturation proceeded, the sockets could be adjusted tighter and, when the full range of a particular socket had been used up, the amputee could be transferred to a smaller size to carry on with conditioning and training. Over the past seven years almost 200 cases with amputation through the thigh have received their early training and conditioning with these temporary adjustable plastic laminate sockets. Since then comparable temporary sockets have been designed and used extensively on $B / K$ amputees five I socket sizes, left and right, satisfying the needs of the bulk of new $\mathrm{B} / \mathrm{K}$ amputees being rehabilitated in Winnipeg.

Limitations of these devices led us quickly into the design of alternative components. A single axis knee-shank unit, which incorporated the Berkeley Pneumatic Swing Phase Control Cylinder and a wedge-disc alignment coupling, were designed and were soon sufficiently useful to serve as temporary devices for $B / K$ and $A / K$ amputees. Although they were initially restricted for use to in-patients, designs soon became dependable enough to use on out-patients. Finally, the devices were sufficiently reliable for use in the final or definitive limb. Then it was possible to initiate amputees into their rehabilitation programmes early with devices which would serve them through all phases of rehabilitation, including the post-rehabilitation phase. A hip fork was designed to permit construction of a modular prosthesis for hip disarticulation amputees also. The three types of prosthese are now available for all stages for $B / K, A / K$ and $H / D$ amputees. The alignment coupling, called the Wedge-DiscAlignment Unit, could be used at different levels in any of the three categories of prostheses. SACH feet were used in all because they are well standardized and provide adequate function. The Hip Disarticulation amputees use the same knee-shank unit as the $A / K$ amputees, and the NorthWestern University Hip Joint is used, a device which is single axis and has alignment adjustability built into it.
Thus it can be seen that only three elements had to be designed to make the system workable for these three levels of amputation: the Winnipeg Single Axis KneeShank Unit, the Wedge-Disc Alignment Unit and the Winnipeg Hip Fork

Recently, a knee disarticulation unit has been designed which is now being introduced for clinical testing. Further, redesign of the Berkeley Pneumatic Swing Phase Control Unit has been completed to improve reliability, simplify manufacturing, and to reduce weight. Improved SACH feet have also been designed. The final version of the SACH foot designed in Winnipeg has a metallic heel and polyurethane reinforcement of the foot around the heel to increase durability of the foot. At the ankle, the shapes of the feet are so organized that a minimum number of shapes permit economy in the design of prefabricated cosmetic covers of fiexible polyurethane.

\section{ASSEMBLY OF A WINNIPEG}

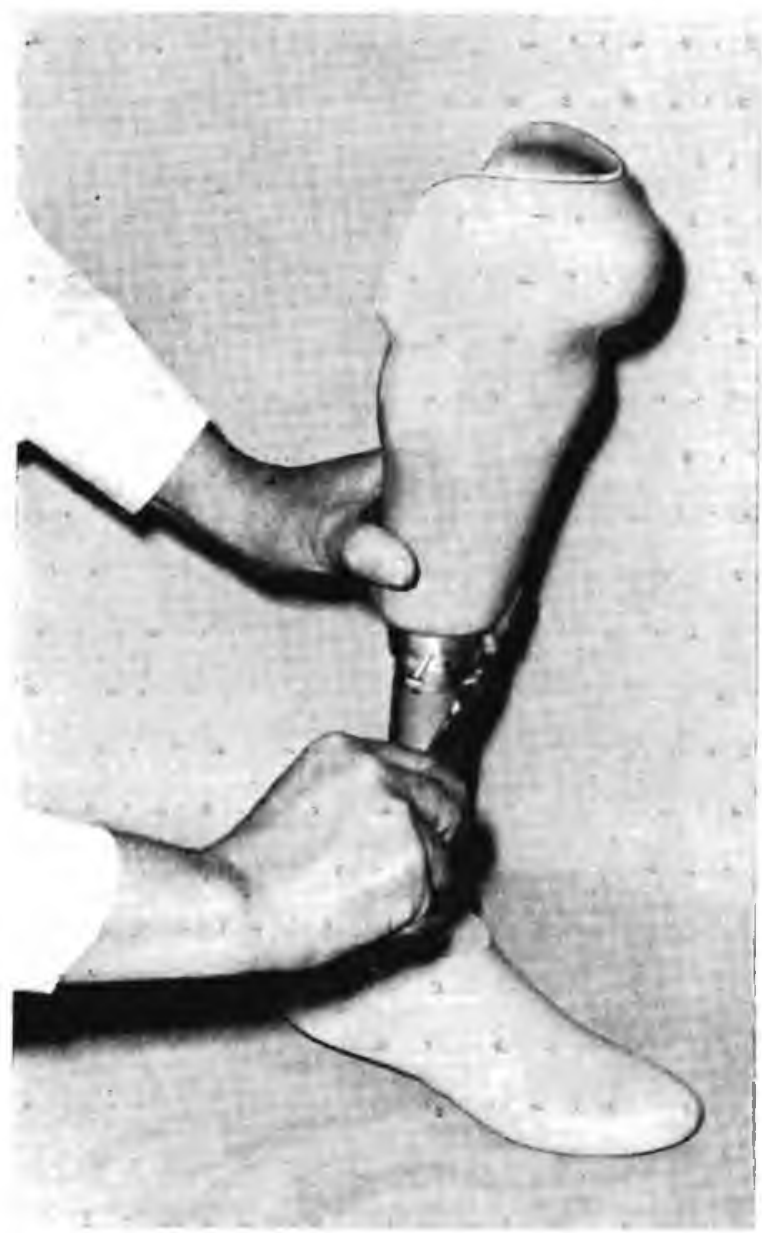

Fig. 1(a) Modular B/K Prosthesis with Wedge-disc Alignment Units, a SACH foot and patellar suspension. 
It is cosmetic restoration, along with non-adjustability of alignment that has been the stumbling block to the introduction of modularized prostheses into clinical services. For the Winnipeg system four cosmetic cover moulds were made which could be matched to six right and six left feet, giving the system one standard calf shape for cosmetic restoration of prostheses for the $\mathrm{B} / \mathrm{K}$ amputees. This single shape has adequately met the needs of approximately 30 per cent of the 300 amputees provided with modular $\mathrm{B} / \mathrm{K}$ prostheses. Programmes to develop a greater range of shapes are currently underway for both the Winnipeg modular system and other modular systems such as the BOCK and BLACHFORD systems being introduced in Germany and England. American developers are also making good headway in the development of cosmetic covers which can be adapted to the Canadian and British systems as well as the American system of modular prostheses now in design. As an alternative, prostheses which do not fall within the cosmetic restoration systems currently available, covers are made of polyester plastic laminates fabricated over sculptured rigid polyurethane foam cast around the pylon structures, or over prefabricated blanks of rigid polyurethane which are sculptured. The plastic shells obtained are separated from the foam after fabrication for installation on the modular limbs. In Winnipeg the thigh sections of hip disarticulation prostheses are covered with a truncated cone of sponge rubber covered with leather. Although soft covers have been designed for the knee-shank unit, the bulk of the $A / K$ amputees receive thin plastic laminate shells for dressing up their prostheses.

A crucial factor in modular prostheses is the capacity to quickly exchange sockets. Deterioration of socket fit due to stump shrinkage has long been a source of trouble for amputees of all levels. The new amputees who pass through the typical rehabilitation services are especially prone to stump shrinkage, often requiring two or three sockets within the first year and a further change after that. In Winnipeg a receptacle system is used to connect the socket to the rest of the prosthesis. The receptacles currently being used are made on a custom basis. Before the socket is completed a plaster extension is cast on to the end of it and a plastic shell fabricated which intimately fits the more proximal parts of the socket and fills out thigh shape for the $A / K$ amputees, or is tailored down for easy containment within the cosmetic cover for the $B / K$ amputees. The base of a receptacle is made flat and at the correct alignment angle for attachment of the rest of the components. The system would be improved if prefabricated attachment brackets were designed to link the socket on to the rest of the prosthesis. For Hip Disarticulation and Hemi Pelvectomy amputees no receptacle is required. For the knee disarticuIation amputee the receptacle is being designed to form an integral part of the knee so that the socket can be plugged into one which is of the correct size.

The $\mathrm{B} / \mathrm{K}$ prosthesis consists of a SACH foot with a Wedgedisc Alignment Unit bolted onto it, a piece of tubing to make up shank length, a second Wedge-disc Alignment Unit bolted on to the socket receptacle and the socket plugged in (Fig. 1). The $\mathrm{A} / \mathrm{K}$ prosthesis consists of a $\mathrm{SACH}$ foot and Wedge-disc Alignment Unit, the Winnipeg Kneeshank Unit, a second Wedge-disc Alignment Unit between the knee and the receptacle, and the receptacle with the socket plugged in (Fig. 2). The $\mathrm{A} / \mathrm{K}$ prosthesis also has a clamp-on valve housing for use with a Hosmar suction socket valve. When belts are used for suspension, attachments are made to the receptacle leaving the socket free. Sockets are bonded to receptacles in the final process of completing the llmb. The Hip Disarticulation Prosthesis consists of a SACH foot, Wedge-disc Alignment Unit on the foot, tubing to make up shank length plugged into the Winnipeg Single Axis Knee-shank Unit, a Wedge-disc Alignment Unit with tubing adapter, tubing to make up thigh length, the Winnipeg Hip Fork with a third Wedgedisc Alignment Unit bolted on, the Northwestern University Hip Joint, and the socket bolted on to the hip joint.

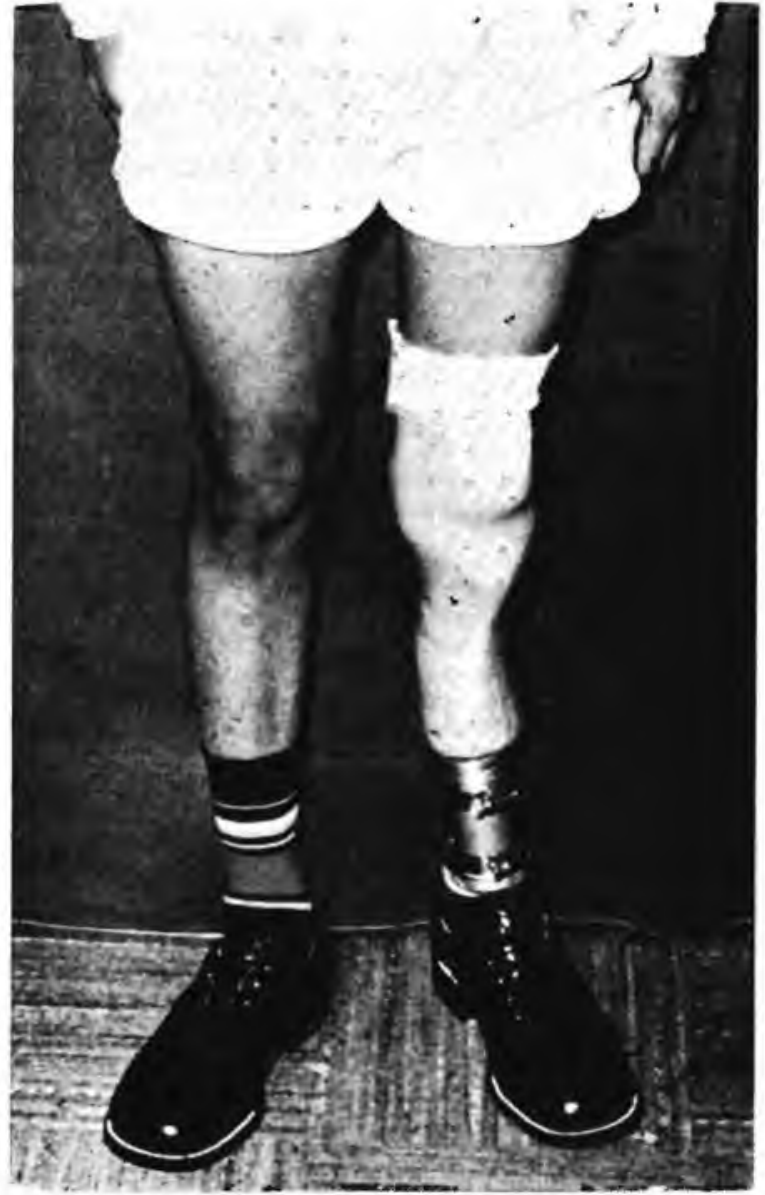

Fig. 1(b) The same type of prosthesis on a $B / K$ amputee, with supracondylar suspension.

The Knee Disarticulation prosthesis consists of a SACH foot, with a Wedge-disc Alignment Unit attached, shank tubing plugged on to a second Wedge-disc Alignment Unit which bolts to the cast aluminium upper shank section, the upper shank section with side arms dovetailed and setscrewed. These fit into clevises laminated into the kneereceptacle section. The socket plugs into the knee-receptacle unit. The knees are pneumatically controlled for the Knee Disarticulation, $\mathbf{A} / \mathbf{K}$, and Hip Disarticulation level prosthesis. Conventional suspension systems are used.

To date, over $300 \mathrm{~B} / \mathrm{K}$ amputees have been provided with modular prostheses in Winnipeg. Approximately 150 A/K amputees have received them. About two dozen $H / D$ amputees use them. No knee disarticulation amputees have yet been fitted with the entire system worked out for them, although half a dozen are wearing a preliminary design which includes BOCK side joints, plastic laminate housings for the attachment of the lower side arms, and the pneumatic swing phase control unit. When upper shank sections are available these will be phased into clinical services.

The future of modular systems is assured for lower extremity amputees and there is no reason to doubt that such systems will be developed for arm and orthotic patients. Use of modular prosthesis will expand as the cosmetic restoration problems are better solved. Meanwhile, those systems which have been proven out clinically should be used for cases and rehabilitation processes which can gain from them. People interested in bringing modularization into clinical services where they have not been used 


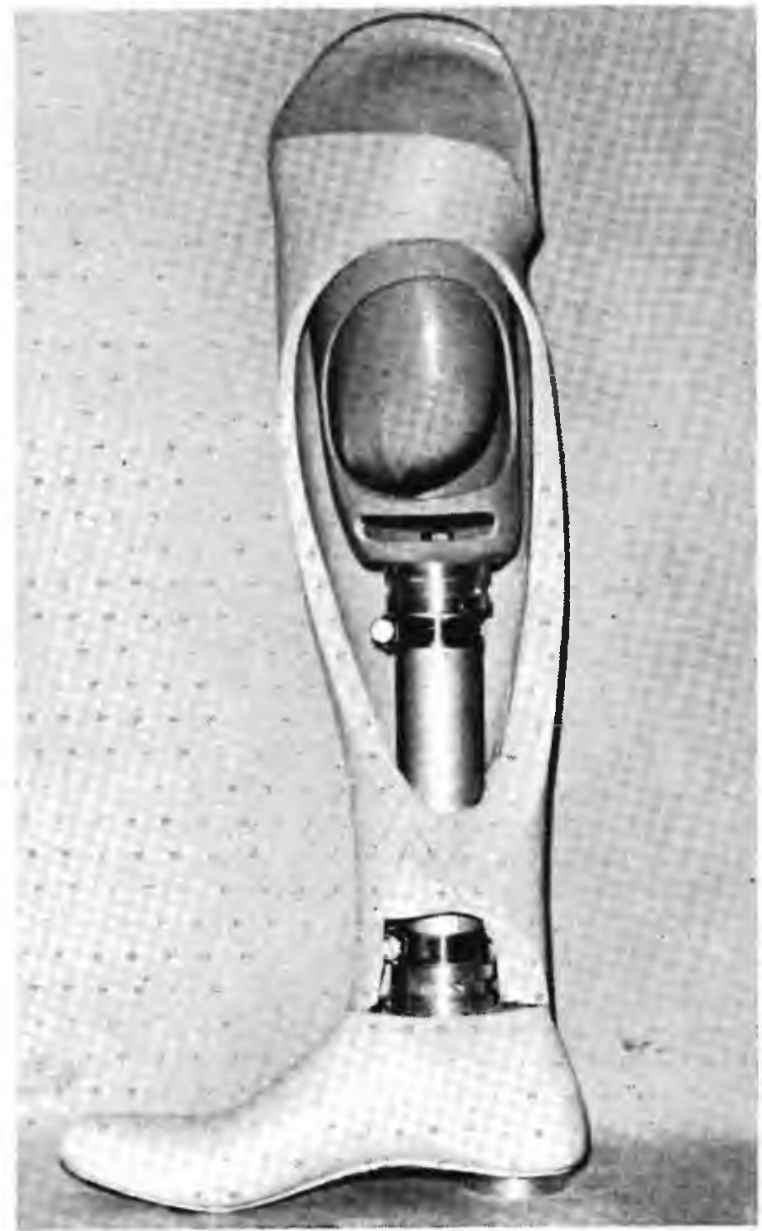

Fig. 1(c) A Supracondylar type of prosthesis cut away to show details of construction: SACH foot, Pylon unit, receptacle for the socket and the socket plugged in.

before should introduce them for handling their new amputees within the rehabilitation setting. As confidence and competence develop, they can, as we did, extend the range of application until all clinical cases can be adequately dealt with using modular components for every phase of care.

Looking ahead to what can be expected of well-developed modular components, these predictions can be made on the basis of Winnipeg experience. Rehabilitation time will be reduced by approximately 50 per cent. Not all of this is due to modularization however. Other factors include early initiation of rehabilitation, greater familiarization with modern amputee handling procedures and closer control over management of the amputee during the early phases of care. But, no doubt, based on experience gained at Winnipeg, it can be safely said that at least a 30 per cent reduction in service time can be attributed to the speed and facility with which adjustments in alignment, adjustments in length and socket replacements can be carried out. The system reported here makes it easy for the prosthetist to provide prostheses quickly as patients enter the rehabilitation stream. (Modifications in alignment and length can be carried out right in clinic if necessary.)

Modularization will permit the development of highly centralized manufacturing and assembly of prostheses by technicians familiar with the system for delivery to the rehabilitation scene within a couple of days. Cosmetic restoration, currently less than adequate, must be brought to a better state if further time gains are to be made in preparating prosthesis. Also a linking system better than the receptacles currently used would speed up fabrication. Currently, fabrication time with the Winnipeg system is approximately half that required for conventional prostheses.

If a method were developed by which socket and leg shape could be defined numerically, the time-consuming processes currently used for these two aspects could be reduced, but, more important, greater systematization and statistical material accumulation would lead to standardization of many shapes now made by laborious hand craft methods. Such a system involves three dimensional shape sensing and reproduction. An example method of sensing is photogrammetry. An object is photographed steriographically, distances between surface points and a reference system are defined in numerical terms. The data can then be used to programme numerically controlled carvers which can produce the shape directly, in mirror image, smaller or larger, on command.

Such mechanization, centralization, modularization and the development of more modern tools for the prosthetist to use at the clinical scene (such as an electrical alignment unit, designed in Winnipeg, which can be used by either prosthetist or amputee to align a foot while the amputee walks) should lead to the prospect of having the prescription for a prosthesis filled within a day or two of prescription, a prosthesis sufficiently adjustable in all important features to permit the prosthetist to bring it into optimum function, and maintain it so, very quickly and easily.

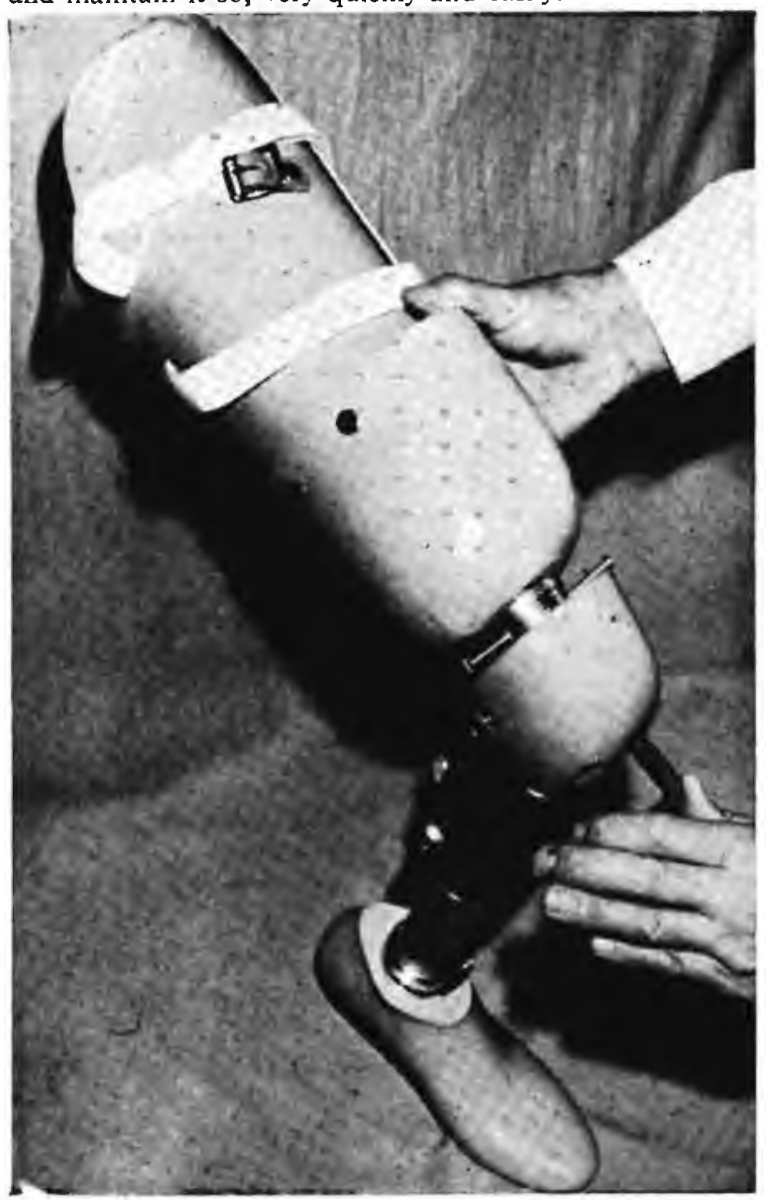

Fig. 2. Assembly of a Winnipeg modular $A / K$ prosthesis with temporary socket for early training and conditioning of an $\mathbf{A} / \mathbf{K}$ amputee. 